\title{
Integration of spatial relationships and temporal relationships in humans
}

\author{
MikaËl Molet, Jeremie Jozefowiez, and Ralph R. Miller \\ State University of New York, Binghamton, New York
}

\begin{abstract}
Three experiments tested human participants on a two-dimensional, computer, landmark-based search task to assess the integration of independently acquired spatial and temporal relationships. Experiment 1 showed that A-B spatial training followed by B-outcome spatial training resulted in spatial integration in such a way that A was effectively associated with the outcome. Experiment 2 showed that A-B spatial and temporal training followed by B-outcome spatial and temporal training resulted in integration that created both spatial and temporal relationships between $\mathrm{A}$ and the outcome. Experiment 3 refuted an alternative explanation, one that is based on decision-making speed, to the temporal-integration strategy that was suggested by Experiment 2 . These results replicate in humans the observations regarding spatial integration made by Sawa, Leising, and Blaisdell (2005) using a spatial-search task with pigeons, and they extend those observations to temporal integration.
\end{abstract}

The sensory preconditioning (SPC) procedure provides strong evidence that subjects can integrate information from separate training episodes through the linkage of common elements (Brogden, 1939). In Phase 1 of a conventional SPC procedure, a stimulus (S1) is forward paired with another stimulus $(\mathrm{S} 2 ; \mathrm{S} 1 \rightarrow \mathrm{S} 2)$. Subsequently, S2 is forward paired with an unconditioned stimulus (US; S2 $\rightarrow$ US) in Phase 2. During testing, a conditioned response appropriate to the US is observed to S1, even though S1 itself is never directly paired with the US. The associative link between $\mathrm{S} 1$ and the US $(\mathrm{S} 1 \rightarrow \mathrm{US})$ suggests that integration of $\mathrm{S} 1 \rightarrow \mathrm{S} 2$ and $\mathrm{S} 2 \rightarrow \mathrm{US}$ associations occurs as a result of superimposing the element that is common to both associations - that is, S2 .

Within the timing literature, Miller and colleagues have presented evidence suggesting that the integration of temporal relationships between paired stimuli can occur in rats and humans (Arcediano, Escobar, \& Miller, 2003, 2005; Matzel, Held, \& Miller, 1988; see also Leising, Sawa, \& Blaisdell, 2007). For instance, Arcediano et al. (2003) used a modified SPC preparation with rats in which they administered $\mathrm{S} 1 \rightarrow \mathrm{S} 2$ pairings in a forward relationship with either a 5-sec gap or no gap between the termination of S1 and the onset of S2 in Phase 1, followed by S2footshock US pairings in a backward relationship (i.e., $\mathrm{US} \rightarrow \mathrm{S} 2$ ) with a 4-sec gap between the termination of the US and the onset of S2 in Phase 2. When tested on S1, rats trained with a 5 -sec gap in Phase 1 showed a large amount of behavioral control by S1, whereas rats trained with no gap in Phase 1 showed less responding to S1. Arcediano et al. hypothesized that the rats had encoded the temporal relationships between S1 and S2 and between the US and $\mathrm{S} 2$, forming two independent temporal maps (including both order and interval) between the paired stimuli. These temporal maps presumably were integrated by superimposing the representation of the common element from the two phases of training (i.e., S2), thereby allowing S1 to predict an immediately impending US in rats that had learned a temporal map with a 5-sec gap in Phase 1 but not in rats that had learned a temporal map with no gap in Phase 1. In the latter case, superimposition of maps would have caused the rats to expect the US to occur simultaneously with S1, a relationship that is not conducive to appreciable behavioral control.

Within the spatial learning literature, research with pigeons has provided evidence highly suggestive of the integration of spatial maps (e.g., Blaisdell \& Cook, 2005; Sawa, Leising, \& Blaisdell, 2005; see also Chamizo, Rodrigo, \& Mackintosh, 2006, for research with rats) in a process similar to the temporal integration that is thought to operate in the timing experiments of Miller and colleagues (Arcediano et al., 2003, 2005; Matzel et al., 1988). Sawa et al. explored the integration of spatial maps in an analogue to Brogden's (1939) SPC procedure, which used a spatial-search task presented on a touch screen to pigeons. On SPC trials, pigeons were presented with two visual landmarks ( $\mathrm{X}$ and $\mathrm{A}$ ) that had a consistent spatial relationship to each other. The pigeons were then reinforced for pecking at an unmarked goal location, which bore a constant spatial relationship to first-order A, which was presented in the absence of X. Subsequent test trials on which $\mathrm{X}$ alone was presented revealed that the pigeons were able to infer a spatial relationship between X and the goal (seemingly, an $\mathrm{X} \rightarrow \mathrm{A} \rightarrow$ goal associative chain). This suggests that pigeons have an ability to combine two independently learned spatial maps into a single spatial map through superposition-

R. R. Miller, rmiller@binghamton.edu 
ing of common elements shared among the maps. Using an open-field procedure with pigeons, moreover, Blaisdell and Cook (2005) reported spatial integration similar to that found by Sawa et al. Research with humans, however, has suggested a contrasting account of performance in comparable situations (Sturz, Bodily, \& Katz, 2006; Sturz, Bodily, Katz, \& Kelly, 2009). Sturz et al. (2006) reported evidence inconsistent with integration of spatial maps in humans when they used a virtual environment open-field search task analogous to Blaisdell and Cook's pigeon foraging task. Sturz et al.'s (2006) data suggested that Blaisdell and Cook's data could reflect the use of an alternative strategy based on generalization from the individual phases of training and a search strategy (e.g., lose-shift) rather than on integration of spatial cognitive maps. Furthermore, Sturz et al. (2009) obtained additional support for their interpretation of human performance in such situations, using a real-world-environment open-field search task that mimicked Sturz et al.'s (2006) virtual-environment task. This account does not appear to be applicable to Sawa et al.'s data described above, however, because Sawa et al.'s touchscreen task was virtually free of potential confounds that may have been present in the open-field procedure. This leaves us with a compelling demonstration of spatial integration by pigeons in a spatial-search task but no clear evidence of spatial integration by either pigeons or humans in an open-field procedure.

One strategy that might be used to assess spatial integration in humans calls for using a preparation that precludes or controls for the alternative strategies mentioned above. Our spatial-learning task was a variant of the touch-screen-equipped monitor task in Sawa et al.'s (2005) experiment with pigeons. The task was presented on a computer screen, which minimized the spatial-searchtask opportunities and controlled for generalization from either phase alone of the sensory-preconditioning task. The former was achieved by allowing participants to make only one choice on each trial. The latter was achieved by having the outcome $(\mathrm{O})$ at test situated at a location different from the one indicated by generalization from either phase alone.

The goals of the present experiments were twofold. The first was to provide evidence for the spatial integration of relationships between two paired stimuli in humans by using a variant of the touch-screen-equipped monitor task used in Sawa et al.'s (2005) experiment with pigeons. The second was to provide the first controlled demonstration of spatial and temporal integration by humans.

\section{EXPERIMENT 1}

Experiment 1 was performed to determine whether humans, like pigeons (Sawa et al., 2005), are able to integrate spatial relationships between paired stimuli acquired during different phases of learning in a spatial-search task.

The participants were assigned to one of two groups: Common Element and No Common Element. The Common Element group assessed the potential integration of spatial relationships sharing a common element (A-B and B-O). The No Common Element group served as a control condition that precluded integration of spatial relationships by presenting entirely different elements in each phase of training ( $\mathrm{A}-\mathrm{B}$ and $\mathrm{C}-\mathrm{O})$. Both groups experienced two observational phases followed by a test trial. Figure 1 shows a summarized design of Experiment 1 and the hypothetical spatial integration for the Common Element group. The participants in the Common Element group were expected to click on a specific square on the basis of the integration of spatial relationships $\mathrm{A}-\mathrm{B}$ and $\mathrm{B}-\mathrm{O}$ - that is, the square above and to the right of A. Participants in the No Common Element group were expected to choose either an association strategy or a generalization strategy. The association strategy was indicated when the chosen square was where B would be expected on the basis of Phase 1 (i.e., behavior

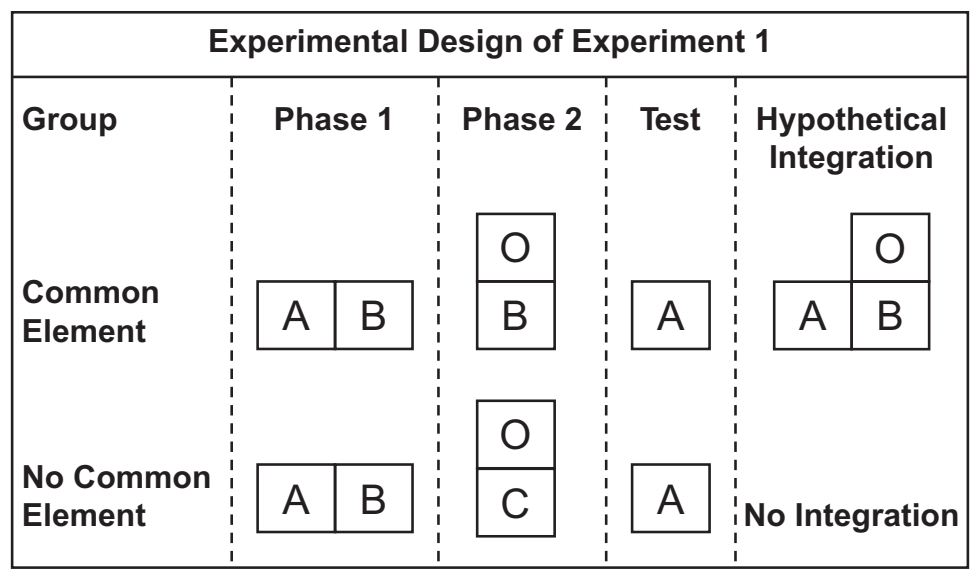

Figure 1. The design and hypothetical spatial representation of Experiment 1. The left side of the figure depicts treatment trials given to the participants. The right side of the figure depicts the spatial representation in the Common Element group that would hypothetically result after spatial integration on the basis of superimposition of the element common to the training phases (i.e., B) and the expected responding based on this integration. 
seemingly was driven by the A-B association alone), and the generalization strategy was indicated when the choice occurred as if the test-trial stimulus was B from Phase 2 rather than $\mathrm{A}$ (i.e., behavior was seemingly driven by the $\mathrm{B}-\mathrm{O}$ association, despite the absence of $\mathrm{B}$ at test).

\section{Method}

Participants and Apparatus. Twenty undergraduate students (10 male, 10 female) at SUNY-Binghamton participated in this study in partial fulfillment of a course requirement. They were randomly assigned to one of two groups: Common Element and No Common Element (both $n \mathrm{~s}=10$ ). Participants performed the experiment using personal computers located in small rooms. The computer in each room was connected to a standard mouse.

Procedure. The task was programmed using Visual Basic 6 (Microsoft). In this task, the computer screen displayed a light-gray $8 \times 8$ grid with black lines (each square was $1 \times 1 \mathrm{~cm}$ ) placed over a dark gray background. Visual stimuli (approximately $0.8 \times 0.8 \mathrm{~cm}$ ) were displayed in select squares of the grid. A blue circle was used as A for all participants. A red cross and a black musical note were used as $\mathrm{B}$ and $\mathrm{C}$, counterbalanced within groups. A green moneybag was used as the outcome O. A black background alone was displayed during intertrial intervals. Instructions (Arial, 18 point) were presented within a gray, rectangular window $(11 \mathrm{~cm}[\mathrm{~h}] \times 29 \mathrm{~cm}[\mathrm{w}])$ on the screen.

Before presentation of the specific instructions for the experiment, a screen informed participants of the general features of the research. The following instructions were presented:

You are about to play a treasure hunt game. You will see several intermittent events on an $8 \times 8$ grid. These events will last for about 3 minutes. During this time, please do not look away from the screen because you might miss seeing an event. When you are ready to begin, please click below.

A gray button with the sentence "click here" was presented at the center bottom of the screen. Participants had to click this with the mouse to continue to the instruction screen that was specific to Phase 1, which read,

You will see the clue randomly placed on the screen, but always at a fixed distance and direction from the clue $\boldsymbol{Ж}$ [or $\mathrm{s}$, counterbalanced within groups]. For the clue $\boldsymbol{O}$, you will have to learn the distance and direction to the clue $\boldsymbol{\mathcal { W }}[$ or $\boldsymbol{\delta}]$. Please do not look away from the screen because you might miss seeing an event. When you are ready to begin, please click below.

In Phase 1, participants were exposed to $10 \mathrm{~A}-\mathrm{B}$ pairings. In all pairings, the onset of A preceded the onset of B, with A and B coterminating. A was displayed for $1.5 \mathrm{sec}$, and B was displayed for $1 \mathrm{sec}$. B was always located one square to the right of A. There was a 1 -sec intertrial interval (ITI) in which the screen was blank (black). The locations of A and B on the grid changed randomly between trials, with the constraint that A did not appear in row 1 (top row) or column 8 (rightmost column), but their relative spatial relationship was unchanged.

At $2 \mathrm{sec}$ after Phase 1, the following instructions appeared on the screen:

Now you will see the clue $\boldsymbol{\mathcal { K }}$ [or $\boldsymbol{s}$, same as 2 nd screen for the Common Element group; different from 2nd screen for the No Common Element group] randomly placed on the screen, but always at a fixed distance and direction from the treasure $\mathbf{8}$ For the clue $\boldsymbol{\mathcal { K }}\left[\begin{array}{ll}\mathrm{J} \\ \mathrm{J}\end{array}\right]$, you will have to learn the distance and direction to the treasure $\mathbf{8}$. Please do not look away from the screen because you might miss seeing an event. When you are ready to begin, please click below.

The Phase 2 procedure was similar to the one described for Phase 1, except that the participants were instructed that they would be observing the spatial relationship between $\mathrm{B}$ and $\mathrm{O}$. B was pre- sented for $1.5 \mathrm{sec}$ on the screen in a random square, except that it did not appear in row 1 or column 1. Again, there were 10 trials. In all pairings, the onset of $\mathrm{B}$ preceded the onset of $\mathrm{O}$ by $0.5 \mathrm{sec}$, with $\mathrm{O}$ and $\mathrm{B}$ coterminating. $\mathrm{O}$ was always located in the square above $\mathrm{B}$. During Phase 2, the two groups differed in the stimuli used. In the No Common Element group, B was replaced with $\mathrm{C}$ in order to omit a common stimulus across Phases 1 and 2 and thus preclude superimposition between relationships during testing. Accordingly, instructions were the same for the two groups, except that B was replaced with $\mathrm{C}$ in the No Common Element group.

At $2 \mathrm{sec}$ after the termination of Phase 2, participants received the following instructions:

You will now be shown the clue $\boldsymbol{0}$. Locate the treasure $\boldsymbol{\text { s }}$ on the grid by using the clue information. To look for the treasure, you will use the mouse to move the cursor on the grid. When the cursor is within the area of the square that you choose, press the left-hand button on the mouse to indicate your choice. Be careful! You can make only one choice. When you are ready to begin, please click below.

Testing consisted of a single test trial. Instructions were consistent across groups. A was presented randomly on the screen but not in row 1 or column 8 .

At the end of the test trial, there was a postexperimental question in which participants were asked how they had made their choice concerning where the treasure was hidden. Immediately after participants answered this question, they were invited to read a debriefing text.

The participants were encouraged through instructions to learn spatial information in each phase of training; however, in no way did the instructions encourage integration of spatial information from the different phases. We expected that the participants would not have a problem remembering the relationships between the stimuli in both observational phases. The purpose of this research was not to address the basis of spatial-information encoding. Rather, our primary interest was to determine whether humans can integrate information from different phases, provided that the separate phases contain some common element.

\section{Results and Discussion}

The majority of participants in the Common Element group ( 9 out of 10) clicked on the square that would be anticipated on the basis of the integration of spatial relationships $\mathrm{A}-\mathrm{B}$ and $\mathrm{B}-\mathrm{O}$ - that is, to say the square above and to the right of A. Conversely, only 1 participant in the No Common Element group $(n=10)$ made the same choice. Participants in the No Common Element group tended to choose either an association strategy (4 participants) or a generalization strategy (5 participants). The association strategy was indicated when the choice was where B would be expected on the basis of Phase 1, and the generalization strategy was indicated when the choice occurred as if the test-trial stimulus was B from Phase 2 rather than A. The difference between the Common Element group and the No Common Element group in choices of the integration square was reliable $\left[\chi^{2}(1,20)=12.80, p<.001\right]$. This result suggests that participants in the Common Element group integrated the separate $\mathrm{A}-\mathrm{B}$ and $\mathrm{B}-\mathrm{O}$ spatial relationships, allowing them to compute an $\mathrm{A}-\mathrm{O}$ spatial relationship, which guided their choice at test. Consistent with this, all participants who exhibited spatial integration indicated on the postexperimental question that they had spatially connected A-B with B-O. Additionally, the location choices that would be expected based on the presumed association and generalization strategies seen in 
the No Common Element group were consistent with the answers given on the postexperimental question.

\section{EXPERIMENT 2}

Experiment 1 provided evidence of spatial integration in humans by using a spatial-search task, thereby replicating Sawa et al.'s (2005) results with pigeons. Perhaps relevant to this demonstration is the demonstration of the integration of independently learned relationships in the temporal domain by humans (Arcediano et al., 2003, 2005). Taking a closer look at the instructions used in Arcediano et al.'s $(2003,2005)$ experiments, however, we cannot safely conclude that the humans were able to integrate two different temporal relationships without explicit instructions to integrate, because the participants were told that "they should remember the relationships between the stimuli in both observational phases because the information from one phase might complete the information from the other phase." In Experiment 2, therefore, we sought a clear demonstration of temporal integration without instructions to integrate. Specifically, this experiment investigated whether spatial and temporal information can be processed and whether such relationships are subject to conjoint integration (i.e., spatial and temporal integration) when they include a common element. We have recently found evidence that rats are able to integrate interval information and that they can use contextual information to select which specific interval information to integrate (Molet, Urcelay, Miguez, \& Miller, in press). The larger issue is whether both human and nonhuman subjects encode and retrieve the where and when of the associated events and whether they can integrate events with a common element. To this end, two groups were initially trained in a fashion similar to that used in
Experiment 1, with the exception that temporal training was provided (see Figure 2). Participants were exposed to a horizontal spatial relationship between A and B with a 3 -sec interstimulus interval, followed by a vertical spatial relationship between $\mathrm{B}$ and $\mathrm{O}$ with a 5 -sec interstimulus interval. If participants in the Common Element group were able to integrate the temporal information from the first and second phases of training on the basis of the common element (i.e., B), the result should be response latencies closer to $8 \mathrm{sec}$ in the Common Element group than in the No Common Element group. Our expectation concerning the spatial response in the Common Element group and the No Common Element group is as stated previously in Experiment 1.

\section{Method}

Participants and Apparatus. Twenty undergraduate students (8 male, 12 female) at SUNY-Binghamton participated in this study in partial fulfillment of a course requirement. They were randomly assigned to one of two groups: Common Element and No Common Element (both $n \mathrm{~s}=10$ ). The data from 1 participant in the Common Element group were discarded because of an error by the experimenter. The apparatus was the same as the one used in Experiment 1. Additionally, in order to evaluate temporal integration, the learning of specific temporal intervals was measured using the latencies of participants clicking on the square of their choice following onset at test of A. Latencies were recorded to the nearest $0.1 \mathrm{sec}$.

Procedure. Before presentation of the specific instructions for the experiment, a screen informed participants of the general features of the research. The text on this screen was similar to that used on the first screen in Experiment 1, except that the experiment duration was said to be "about 5 minutes" (as opposed to "about 3 minutes" in Experiment 1). In Phase 1, the following instructions appeared on the computer screen:

On each trial, you will see the clue randomly placed on the screen. Upon each presentation, it will be followed after a fixed

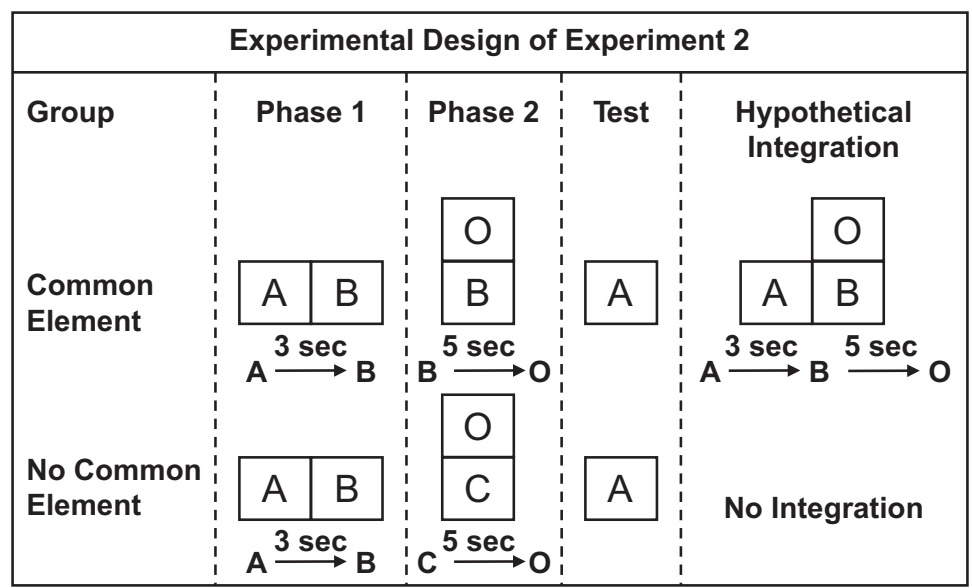

Figure 2. The design and hypothetical spatial and temporal representation of Experiment 2. The left side of the figure depicts the spatial and temporal training phases given to the participants. The ISI between the onset of $A$ and the onset of B was $3 \mathrm{sec}$, and the ISI between the onset of B or C and the onset of $O$ was $5 \mathrm{sec}$. The right side of the figure presents the spatial and temporal representation in the Common Element group that would hypothetically result if participants integrate spatial and temporal information through superimposition of the element common to the training phases (i.e., B) and the expected responding based on these integrations (i.e., where and when the outcome should appear on the grid). 
interval by the clue $\boldsymbol{\mathcal { X }}$ [or $\boldsymbol{\Omega}$, counterbalanced within groups] at a fixed distance and direction from the clue $\mathbf{0}$. For the clue you will have to learn the delay, distance, and direction to the clue $\boldsymbol{\mathcal { W }}\left[\begin{array}{ll}\mathrm{s} & \boldsymbol{\Xi}\end{array}\right]$. Counting may help you learn the timing. Please do not look away from the screen because you might miss seeing an event. When you are ready to begin, please click below.

In Phase 1, participants were exposed to $10 \mathrm{~A}-\mathrm{B}$ pairings. In all pairings, the onset of A preceded the onset of $B$, with the 4-sec presentation of $\mathrm{A}$ and the 1-sec presentation of $\mathrm{B}$ coterminating; hence, the ISI between the onset of A and the onset of B was $3 \mathrm{sec}$. B was always located one square to the right of A. There was a 1-sec ITI during which the screen was blank (black). The locations of A and B on the grid changed randomly between trials (with the constraint that A did not appear in row 1 or column 8), but the spatial relationship was unchanged. Participants were instructed that the delay, distance, and direction of the two clues were important.

At $2 \mathrm{sec}$ after Phase 1, the following instructions appeared on the screen:

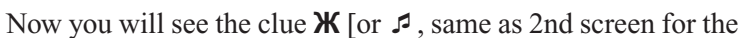
Common Element group; different from 2nd screen for the No Common Element group] randomly placed on the screen. Upon each presentation, it will be followed after a fixed interval by the treasure $\boldsymbol{\Xi}$ at a fixed distance and direction from the clue $\boldsymbol{W}$ $\left[\begin{array}{ll}\text { or } & \boldsymbol{J}\end{array}\right]$. For the clue $\boldsymbol{Ж}\left[\begin{array}{ll}\text { or } & \boldsymbol{\Omega}\end{array}\right]$, you will have to learn the delay, distance, and direction to the treasure $\mathbf{\bullet}$. Counting may help you learn the timing. Please do not look away from the screen because you might miss seeing an event. When you are ready to begin, please click below.

In Phase 2, the second spatial relationship was introduced $2 \mathrm{sec}$ after the participant clicked. The procedure was similar to that used in Phase 1, except that the participants were instructed that they would have to observe the spatial and temporal relationships between B and O. B was presented on the screen, avoiding its placement in row 1 or column 1. As in Experiment 1, there were 10 trials. In all pairings for the Common Element group, the onset of B - now $6 \mathrm{sec}$ in duration - preceded the onset of the 1-sec presentation of $\mathrm{O}$, with $\mathrm{B}$ and $\mathrm{O}$ coterminating; hence, the ISI between the onset of $\mathrm{B}$ and the onset of $\mathrm{O}$ was $5 \mathrm{sec}$. O was always located one square above B. B was replaced with C for the No Common Element group, in order to avoid there being a common element between Phase 1 and Phase 2. Accordingly, instructions were the same across groups, except that B was replaced with $\mathrm{C}$ in the instructions for the No Common Element group.

At $2 \mathrm{sec}$ after Phase 2, the participants received the following instructions:

You will now be shown the clue 0 . Locate where and when the treasure $\boldsymbol{\Xi}$ should appear on the grid by using the clue information. To look for the treasure, you will use the mouse to move the cursor on the grid. When the cursor is within the square that you choose, press the left-hand button on the mouse to indicate your choice at the moment you anticipate the treasure. Counting may help you with the timing. Be careful! You can make only one choice. When you are ready to begin, please click below.

Testing consisted of a single test trial. Instructions were the same across the two groups. A was presented randomly on the screen, except that it did not appear in row 1 or column 8.

At the end of the test trial, there were two brief postexperimental questions in which participants were asked (1) how they had made their choice concerning where the treasure was hidden, and (2) how they had made their choice concerning when they expected the treasure. After the participants answered those questions, they were invited to read a debriefing text.

Notably, the participants were encouraged through instructions to learn spatial and temporal information in each phase of training. But the instructions did not encourage integration of spatial and temporal information from different phases.

\section{Results and Discussion}

The majority of participants in the Common Element group ( 7 out of 9) responded to the square that would be anticipated on the basis of the integration of spatial relationships $\mathrm{A}-\mathrm{B}$ and $\mathrm{B}-\mathrm{O}$ - that is, the square above and to the right of A. In the No Common Element group $(n=10)$, no participant chose this square; the choices suggested the association and generalization strategies (4 participants and 6 participants, respectively). The difference between the Common Element group and the No Common Element group in choice of the integration square was reliable $\left[\chi^{2}(1,19)=11.45, p<.001\right]$. This result was highly similar to that of Experiment 1, and it suggests that participants in the Common Element group integrated the A-B and $\mathrm{B}-\mathrm{O}$ spatial relationships, allowing them to arrive at an $\mathrm{A}-\mathrm{O}$ spatial relationship that guided their choice at test.

For the Common Element group, we expected that presentation of $\mathrm{A}$ would activate a representation of $\mathrm{B}$ occurring $3 \mathrm{sec}$ after the onset of A. This B representation in turn was expected to activate a representation of $\mathrm{O}$ occurring $5 \mathrm{sec}$ after the onset of the B representation (i.e., an integration strategy). The temporal expectation for participants in the No Common Element group would be based on either Phase 1 alone (i.e., an association strategy) or Phase 2 alone (i.e., a generalization strategy), resulting in latencies near 3 or $5 \mathrm{sec}$, respectively. Figure 3 depicts group mean response latencies for participants clicking on the square of their choice (independent of the choice being correct) after onset of the presentation of the test A. As can be seen, latency to respond in the Common Element group was closer to $8 \mathrm{sec}$ (the sum of 3 and $5 \mathrm{sec}$ ) than was the latency to respond in the No Common Element group.

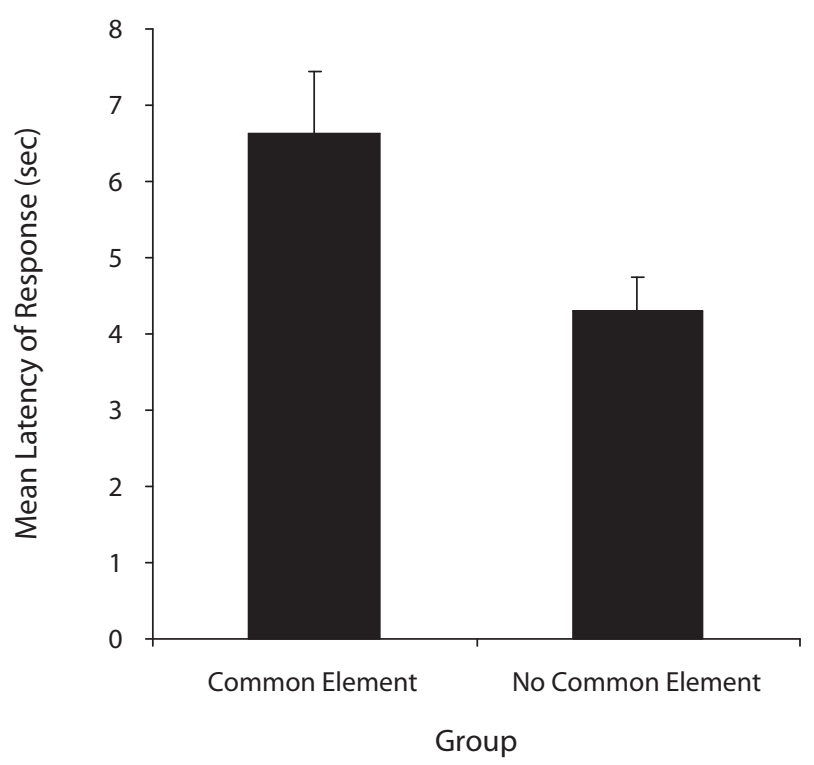

Figure 3. The results of Experiment 2. Mean latency of response (i.e., expectation of the outcome) for the stimulus-A test trial in the Common Element and No Common Element groups. Error bars represent the standard errors of the means. Temporal integration is evidenced in the higher latency to respond during stimulus-A presentation in the Common Element group than in the No Common Element group. 
The difference in latencies between the Common Element group and the No Common Element group was reliable [Mann-Whitney $U(9,9)=15, p=.02$ ]; thus, participants in the Common Element group seemingly integrated temporal information from the first and second phases of training (ISI of $3 \mathrm{sec}$ for A-B and ISI of $5 \mathrm{sec}$ for B-O, respectively) to predict when $\mathrm{O}$ should appear. Additionally, all participants who exhibited behavior that was suggestive of spatial and temporal integration indicated in their answers to the postexperimental questions that they had tried to connect A-B with B-O both spatially and temporally; and all participants who used association and generalization strategies indicated in their answers to the postexperimental questions that they had made their choice on the basis of Phase 1 or Phase 2, respectively.

To recapitulate, Experiment 2 showed that A-B spatial and temporal training followed by $\mathrm{B}-\mathrm{O}$ spatial and temporal training resulted in integration that created both spatial and temporal relationships between $\mathrm{A}$ and $\mathrm{O}$.

\section{EXPERIMENT 3}

In Experiment 2, we hypothesized that if participants in the Common Element group were able to integrate the temporal information from the first and second phases of training on the basis of the common element (i.e., B), then response latencies would be closer to $8 \mathrm{sec}$ in the Common Element group than in the No Common Element group. There is an alternative interpretation of this difference, however. It is possible that the difference in mean response latencies between the two groups was due to the difference in the amount of time required to integrate purely spatial relationships and to decide in which square the treasure was hidden. If this were the case, then a difference in reaction times should still be detectable in a simple experiment in which participants are not asked to indicate when the treasure will appear. If no difference in responding was observed between groups in such an experiment, however, then we could reject this alternative and conclude that the higher latency of response to $\mathrm{A}$ in the Common Element group was the result of integration of temporal relationships. Experiment 3 was conducted to address this issue. The design of the experiment was identical to the design of Experiment 2, with the exception that only spatial integration was required. The instructions were accordingly adapted.

\section{Method}

Participants and Apparatus. Twenty undergraduate students (11 male, 9 female) at SUNY-Binghamton participated in this study in partial fulfillment of a course requirement. The apparatus was the same as that used in Experiment 2.

Procedure. The procedure was nearly the same as described for Experiment 2. In order to compare the time to make a decision between the Common Element group and the No Common Element group (both $n \mathrm{~s}=10$ ), we recorded each participant's reaction time to make a choice of location in testing. The instructions for the first three screens were the same as those used in Experiment 1. The screen shown immediately prior to testing read as follows:

You will now be shown the clue 0 . Locate as quickly as possible the treasure $\boldsymbol{\Xi}$ on the grid by using the clue information. To look for the treasure, you will use the mouse to move the cursor on the grid. When the cursor is within the area of the square that you choose, press the left-hand button on the mouse to indicate your choice. Be careful! You can make only one choice. When you are ready to begin, please click below.

\section{Results and Discussion}

The majority of participants in the Common Element group (8 out of 10) responded to the square that was appropriate given integration of spatial relationships A-B and $\mathrm{B}-\mathrm{O}$ (i.e., a square above and to the right of $\mathrm{A}$ ). In contrast, only 1 participant in the No Common Element group $(n=10)$ chose the integration square; the remaining choices were consistent with the association and generalization strategies (5 participants and 4 participants, respectively). The difference between the Common Element group and the No Common Element group in choice of the integration square was reliable $\left[\chi^{2}(1,20)=9.9, p<.01\right]$. On the postexperimental question, all participants showing spatial integration indicated that they had spatially connected A-B with B-O; and all participants who seemingly used association or generalization strategies gave answers that were consistent with their apparent strategies. The results of this experiment thus replicated the spatialintegration effect found in Experiments 1 and 2.

Figure 4 depicts group mean response latencies measured from the onset of test A. As can be seen, there was no appreciable difference in time to make a choice between the two groups. A Mann-Whitney $U$ test failed to detect a difference between the Common Element and No Common Element groups $[U(10,10)=45, p=.74]$. Because the relevant conclusion of this analysis was that there was no difference between the Common Element and No Common Element groups, we conducted a test to determine whether these mean response latencies were statistically equivalent

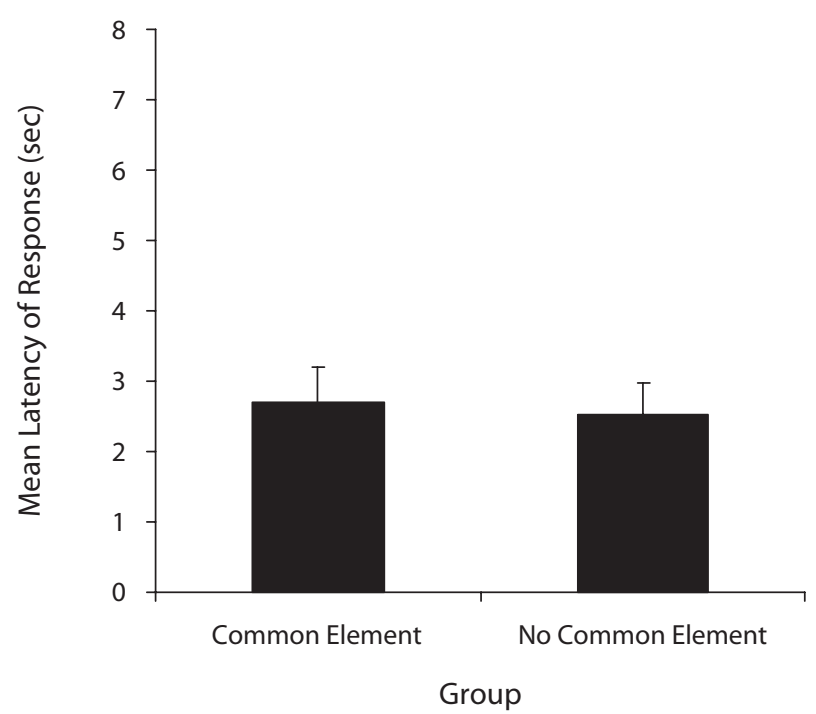

Figure 4. The results of Experiment 3. Mean latency of response for the stimulus-A test trial in the Common Element and No Common Element groups. Error bars represent the standard errors of the means. 
across groups. The test we used was a confidence interval equivalence test (Rogers, Howard, \& Vessey, 1993). To perform this test, we selected an equivalence interval of $10 \%$ around the mean of the Common Element group. The resultant equivalence interval had a lower limit of 2.421 and an upper limit of 2.959. To be considered equivalent, the standardized difference between the observed group means should fall between the upper and lower limits of the equivalence interval. To standardize the difference between the group means, we calculated $z$ values using the formula $\left(\left[M_{1}-M_{2}\right]-\left[E I_{\mathrm{U}}\right.\right.$ or $\left.\left.E I_{\mathrm{L}}\right]\right) / s_{M_{1}-M_{2}}$, where $M_{1}$ and $M_{2}$ represent the means of the Common Element group and the No Common Element group, respectively; $E I_{\cup}$ and $E I_{\mathrm{L}}$ represent the upper limit and lower limit, respectively, of the equivalence interval; and $s_{M_{1}-M_{2}}$ represents the standard deviation of the difference between $M_{1}$ and $M_{2}$. The resultant $z$ values of 0.64 and -0.14 were compared with the critical value $\left(z_{.05}=1.65\right)$. Because the highest $z$ value (0.64) was lower than the critical value, we concluded that the distributions were equivalent (see Rogers et al., 1993, for a detailed description of confidence interval equivalence testing); that is, time to make a choice was statistically equivalent between the two groups. We thus find it implausible that between-group differences in decisionmaking speeds contributed appreciably to the large difference in response latencies in Experiment 2. In conclusion, the difference in mean latencies observed in Experiment 2 was likely due to temporal integration of the two phases of training, causing Common Element group participants to attempt to approximate $8 \mathrm{sec}$.

\section{GENERAL DISCUSSION}

The results of these three experiments provide evidence that human subjects have the ability to combine two independently learned spatial relationships into a unified, higher order relationship by superimposing the relationships in memory on the basis of common elements shared between the relationships; that is, A-B spatial training followed by $\mathrm{B}-\mathrm{O}$ spatial training resulted in spatial integration that connected $\mathrm{A}$ with $\mathrm{O}$. Moreover, this occurred without any explicit instructions to integrate the spatial relationships. This suggests that integration of spatial relationships is a default strategy. Notably, the instructions in the present experiments encouraged learning of spatial and temporal relationships but not their integration. The present results are consistent with the results of Sawa et al. (2005) with pigeons and Chamizo et al. (2006) with rats.

It is noteworthy that integration of cognitive relationships has previously been extended into the temporal domain (Matzel et al., 1988; Savastano \& Miller, 1998). Investigating this commonality between the spatial and the temporal, Experiment 2 constitutes the first controlled demonstration of conjoint integration of cognitive temporal and spatial relationships. Participants were exposed to a horizontal spatial relationship between A and B with a 3 -sec ISI, followed by a vertical spatial relationship between $\mathrm{B}$ and $\mathrm{O}$ with a 5-sec ISI. Our results showed that $\mathrm{A}-\mathrm{B}$ spatial and temporal training followed by $\mathrm{B}-\mathrm{O}$ spa- tial and temporal training resulted in spatial and temporal integration that created both spatial and temporal relationships between $\mathrm{A}$ and $\mathrm{O}$.

Another seeming example of animals using spatial and temporal information was provided by Molet et al. (in press), in which rats were able to encode and retrieve the where and when of the associated events and could integrate events with a common element (see also Cheng, Spetch, \& Miceli, 1996, for similar results with pigeons). Previous data in the literature supportive of temporal integration (Arcediano et al., 2003, 2005; Molet et al., in press) and of spatial integration (Chamizo et al., 2006; Sawa et al., 2005), in conjunction with our present results, support the hypothesis that the processes that integrate information concerning separate but related associations when using spatial information work in a way that is similar to the processes involving temporal information in experiments designed in the framework of the temporal coding hypothesis (see Savastano \& Miller, 1998, for a summary).

Previous research with humans, however, has provided a contrasting account of performance in open-field procedure. Sturz et al. (2006) reported evidence that was inconsistent with integration of spatial maps in humans when they used a virtual-environment open-field search task analogous to Blaisdell and Cook's (2005) pigeon open-field procedure. Sturz et al.'s (2006) data suggested that the participants' performance could reflect use of an alternative strategy based on generalization from the individual phases of training and a search strategy (e.g., lose-shift) rather than on integration of spatial cognitive maps. Furthermore, Sturz et al. (2009) obtained additional support for their interpretation of human performance in such situations, using a real-world-environment open-field search task that mimicked Sturz et al.'s (2006) virtual-environment task. This account does not appear to be applicable to Sawa et al.'s (2005) data, however, because Sawa et al.'s touch-screen task was virtually free of the opportunity for these alternative strategies, which were present in the open-field procedure. For this reason, we used a two-dimensional, computerized, landmarkbased, single-choice search task with humans, which minimized the spatial-search-task opportunities and controlled for generalization from either phase alone of the sensory-preconditioning task. The former was achieved by allowing participants to make only one choice on each trial. The latter was achieved by having the outcome at test situated at a location different from the one indicated by generalization from either phase alone. These potentially critical differences between the two studies appear to have circumvented accounts based on search patterns, such as the account Sturz et al. had raised as an alternative to spatial integration by humans.

\section{AUTHOR NOTE}

M.M. is currently at the Université Charles de Gaulle-Lille 3 (Villeneuve d'Asq, France). J.J. is currently at the Universidade do Minho (Braga, Portugal). This research was supported in part by a postdoctoral fellowship from the Fyssen Foundation to M.M. We thank Aaron Blais- 
dell, Mario Laborda, Bridget McConnell, Gonzalo Miguez, Cody Polack, and James Witnauer for their comments on earlier versions of the manuscript, and Olivier Lefebvre for discussions of the experimental task. The authors also thank three anonymous reviewers for their comments on an earlier version. Correspondence concerning this article should be addressed to R. R. Miller, Department of Psychology, SUNY at Binghamton, Binghamton, NY 13902-6000 (e-mail: rmiller@binghamton.edu).

\section{REFERENCES}

Arcediano, F., Escobar, M., \& Miller, R. R. (2003). Temporal integration and temporal backward associations in humans and nonhuman subjects. Learning \& Behavior, 31, 242-256.

Arcediano, F., Escobar, M., \& Miller, R. R. (2005). Bidirectional associations in humans and rats. Journal of Experimental Psychology: Animal Behavior Processes, 31, 301-318.

Blaisdell, A. P., \& Cook, R. G. (2005). Integration of spatial maps in pigeons. Animal Cognition, 8, 7-16.

Brogden, W. J. (1939). Sensory pre-conditioning. Journal of Experimental Psychology, 25, 323-332.

Chamizo, V. D., Rodrigo, T., \& Mackintosh, N. J. (2006). Spatial integration with rats. Learning \& Behavior, 34, 348-354.

Cheng, K., Spetch, M. L., \& Miceli, P. (1996). Averaging temporal duration and spatial position. Journal of Experimental Psychology: Animal Behavior Processes, 22, 175-182.

Leising, K. J., Sawa, K., \& Blaisdell, A. P. (2007). Temporal integra- tion in Pavlovian appetitive conditioning in rats. Learning \& Behavior, 35, 11-18.

Matzel, L. D., Held, F. P., \& Miller, R. R. (1988). Information and expression of simultaneous and backward associations: Implications for contiguity theory. Learning \& Motivation, 19, 317-344.

Molet, M., Urcelay, G. P., Miguez, G., \& Miller, R. R. (in press). Using context to resolve temporal ambiguity. Journal of Experimental Psychology: Animal Behavior Processes.

Rogers, J. L., HowArd, K. I., \& Vessey, J. T. (1993). Using significance tests to evaluate equivalence between two experimental groups. Psychological Bulletin, 113, 553-565.

Savastano, H. I., \& Miller, R. R. (1998). Time as content in Pavlovian conditioning. Behavioural Processes, 44, 147-162.

Sawa, K., Leising, K. J., \& Blaisdell, A. P. (2005). Sensory preconditioning in spatial learning using a touch screen task in pigeons. Journal of Experimental Psychology: Animal Behavior Processes, 31, 368-375.

Sturz, B. R., Bodily, K. D., \& Katz, J. S. (2006). Evidence against integration of spatial maps in humans. Animal Cognition, 9, 207-217.

Sturz, B. R., Bodily, K. D., Katz, J. S., \& Kelly, D. M. (2009). Evidence against integration of spatial maps in humans: Generality across real and virtual environments. Animal Cognition, 12, 237-247.

(Manuscript received May 4, 2009; revision accepted for publication July 6,2009 .) 